\title{
Position Coordination of a Linear Teleoperation System with Constant Time Delay
}

\author{
Kamran Razi, S. MJ. Yazdanpanah, Saeed Shiry Ghidary
}

\begin{abstract}
A linear time invariant controller design for a bilateral teleoperation of a pair of $\mathrm{N}-\mathrm{DOF}$ linear robotic systems under constant time delay is presented. This framework uses position and velocity signals together with the force signals to compensate human/environment disturbances and achieve position coordination in both free motion and contact tasks. In this approach, by using Parseval's identity passivity analysis is done in the frequency domain to render teleoperator passive. In passifying position and velocity feedback terms it is no need to consider the dynamics of the robotic systems as long as they satisfy Euler-Lagrangian dynamic system equation[1] . This fact simplifies the analysis and design routine. However it is shown that when force feedback is applied, gaining advantage of controller and robot dynamics decoupling is no longer available. Simulation is performed to illustrate the proposed algorithm and clarify some points in the design procedure.
\end{abstract}

\section{INTRODUCTION}

A telerobotic system is aimed at transferring a sense of remote task to the operator as he feels if he is performing that task directly. To do this, the teleoperator, consisting of master and slave robots, their controls, and the communication between them (see Fig.1), should be transparent, i.e. it has to transfer the mechanical impedance to the operator with the minimum distortion in the frequency of interest [4]. Also, this goal may be interpreted as the equality between two force signals, $F_{1}$ and $F_{2}$, and two position signals, $q_{1}$ and $q_{2}$. So, in the most simple case, 1DOF master and slave robots, we deal with a multivariable control problem. Moreover, there are two uncertain subsystems namely human and environment interfering in the dynamic properties of the overall system. Fortunately, human operator proved to be passive in working conditions and assumption of coupling with a passive environment makes the controlled system applicable to a large class of tasks. A control system to be considered safe should be robust to these subsystems. In some works [5 and 8], human and the environment are modeled by a linear mass-damperspring impedance with unknown gains but belong to a certain bounds. Some other works [16] presumes environment and human operator passive and makes use of Llewellyn criteria or infinity norm of the scattering matrix of

K.Razi (email: k.razi@ece.ut.ac.ir) and SMJ. Yazdanpanah (email: yazdan@ut.ac.ir) are with the Conrol and Intelligent Processing Center of Excellence (CIPCE), University of Tehran, Iran. S. Sh. Ghidary (email: shiry@aut.ac.ir) is with the department of computer engineering and information technology, Amirkabir university of technology, Tehran, Iran. the teleoperator to guarantee the stability of the system. These approaches are applicable in the case of linear systems.

Position coordination is an important concern in designing such systems. In scattering-based approaches extra control loops should be added to ensure the position tracking because of the lack of explicit position feedback [1]

Although a real world application of a teleoperation system consists of multi-DOF robotic systems, many contributing works make use of a 1-DOF linear model [4, 16 , and 10] to focus the discussion to the important issues in a teleoperation system rather than multivariable and nonlinear control. $\mathrm{H}_{\infty}$ robust control approaches have an advantage in that they are easily applicable to multi-DOF cases $\left[6,13,14\right.$, and 15]. Unlike $\mathrm{H}_{\infty}$ approaches, some other works choose a pre-specified control structure and adjust the parameters to obtain passivity of the teleoperator and performance [1,2, and 3].

As a case in point, Lee et al in [2], firstly, transfer the problem of the teleoperation of two n-DOF robotic systems to control of two decoupled n-DOF systems namely? and? The first system is controlled with a PD controller to achieve position coordination of the overall system and?? to shape the dynamics of the overall system by use of impedance control approaches. Their work is generalized in [3] for an Euler-Lagrangian nonlinear systems. In [1], they apply this approach to the case of time-delayed teleoperation with nonlinear dynamics. They use just position information in the feedback loop. So, position coordination can not be achieved in the presence of human and environment disturbances forces. Mentioned works are similar in that passive implementation of the controller is done for the control terms individually. Clearly, this would simplify the control design task. This paper is in the line of these works.

We assign a pre-specified control structure and set conditions for the passivity for its terms. In addition to PD control, a force compensation term is added to achieve position coordination in both cases of free motion and contact tasks.

Manipulator's motion can be divided into three different phases, free motion, contact motion, and the transition between them. There is large number of papers considering the first two issues, but the complexity which the variety of the environments adds in modeling makes the transition discussion difficult. Important issues in contact transition like impact forces and number and amplitude of bounces are 
discussed in [7]. Ni et al discuss these issues in [8] for a 1-DOF linear teleoperation system and linearly modeled human and environment. Other works prefer to bypass this problem by some practical solutions like adding damper impedance to the system as reported to be the most important factor in stability of the contact transition [9]. In this work, the additional terms added to passify the controlled system are mainly dissipative ones, so it is sensible to leave the performance of contact tasks to the simulation.

\section{MODELLING}

Consider a teleoperation system which consists of two NDOF robotic systems as the master and slave robots governed by two linear systems as follows,

$$
\begin{aligned}
& M_{1} \ddot{q}_{1}(t)=T_{1}(t)+F_{1}(t) \\
& M_{2} \ddot{q}_{2}(t)=T_{2}(t)+F_{2}(t)
\end{aligned}
$$

where $q_{1}, q_{2} \in \Re^{n}$ are the position configurations, $F_{1}, F_{2} \in \mathfrak{R}^{n}$ represent the human and environment force respectively, $T_{1}, T_{2} \in \mathfrak{R}^{n}$ are control signals, and $M_{1}, M_{2} \in \Re^{n \times n}$ are the positive-definite $(P D)$ inertia matrices. Each of the robots can be assumed as an Euler-Lagrangian dynamic system where Coriolis matrix is zero. It is also known that the controller passivity, i.e.

$$
\int_{0}^{t}\left[T_{1}^{T}(\theta) \dot{q}_{1}(\theta)+T_{2}^{T}(\theta) \dot{q}_{2}(\theta)\right] d \theta \leq c^{2} \quad \forall t \geq 0
$$

implies energetic passivity of the teleoperation system (), i.e.

$$
\int_{0}^{t}\left[F_{1}^{T}(\theta) \dot{q}_{1}(\theta)+F_{2}^{T}(\theta) \dot{q}_{2}(\theta)\right] d \theta \leq-d^{2} \quad \forall t \geq 0
$$

This property is so beneficial in that one can just ensure the passivity of the teleoperator without concerning the nonlinear dynamics of the robots as long as it is EulerLagrangian dynamic. However, when force feedback is applied to compensate the effects of $F i, i=1,2$ to achieve position compensation, this decoupling in design can not be used and the dynamics of the teleoperator should also be considered.

\section{CONTROL DESIGN}

Position error-based control used in [1] ensures position coordination just in free motion (when $F i=0, i=1,2$ ). Here, force feedback is also added to provide system with position coordination in both cases of free motion and contact tasks. Hence, the overall control is designed to be

$$
\begin{aligned}
T_{1}(t)= & \overbrace{K_{v}\left(\dot{q}_{2}\left(t-\tau_{2}\right)-\dot{q}_{1}(t)\right)}^{\text {Derivative term }}+\overbrace{K_{p}\left(q_{2}\left(t-\tau_{2}\right)-q_{1}(t)\right)}^{\text {Proportional term }} \\
& +\underbrace{}_{\text {Compensation term }}+K_{f 11} F_{1}(t)+K_{f 12} F_{2}\left(t-\tau_{2}\right) \\
T_{2}(t)= & \underbrace{-K_{1 d_{0}} \dot{q}_{1}(t)-\sum_{j=1}^{4} K_{1 d_{j}} \dot{q}_{1}(t)}_{K_{v}\left(\dot{q}_{1}\left(t-\tau_{1}\right)-\dot{q}_{2}(t)\right)}+\overbrace{K_{p}\left(q_{1}\left(t-\tau_{1}\right)-q_{2}(t)\right)}^{\text {Proportionalion terms }} \\
& +\underbrace{+K_{f 21} F_{1}\left(t-\tau_{1}\right)+K_{f 22} F_{2}(t)}_{\text {Compensation term }} \underbrace{-K_{2 d_{0}} \dot{q}_{2}(t)-\sum_{j=1}^{4} K_{2 d_{j}} \dot{q}_{2}(t)}_{\text {Dissipation terms }}
\end{aligned}
$$

where $K_{v}, K_{p}$ are the symmetric and positive-definite PD gains and $K_{i d j}$ are four dissipation gains for each robot. One of them is to passify the effects of proportional control action and the other three to passify the effects of the force compensation term. $\tau_{1}, \tau_{2}$ are the constant communication delays.

Design of the control consists of two steps. First, passify the effects of the PD control gains. In this step decoupling of the controller and robotic manipulator is utilized to simplify the design procedure. In the second step force compensation term become passive. As will be shown this requires incorporation of the system dynamics as well as PD control actions.

The power generated by the controller is given by

$$
\begin{aligned}
P_{c}(t) & =T_{1}^{T} \dot{q}_{1}(t)+T_{2}^{T} \dot{q}_{2}(t) \\
& =P_{p d}(t)+P_{f}(t)
\end{aligned}
$$

in which $p_{p d}$ and $p_{f}$ are the power associated with the PDcontrol and force compensation terms respectively and are defined by

$$
\begin{aligned}
& P_{P D}(t)= \\
& \dot{q}_{1}(t) \cdot\left[K_{v}\left(\dot{q}_{2}\left(t-\tau_{2}\right)-\dot{q}_{1}(t)\right)+K_{p}\left(q_{2}\left(t-\tau_{2}\right)-q_{1}(t)\right)-K_{1 d_{0}} \dot{q}_{1}(t)\right] \\
& +\dot{q}_{2}(t) \cdot\left[K_{v}\left(\dot{q}_{1}\left(t-\tau_{2}\right)-\dot{q}_{2}(t)\right)+K_{p}\left(q_{1}\left(t-\tau_{1}\right)-q_{2}(t)\right)-K_{2 d_{0}} \dot{q}_{2}(t)\right] \\
& P_{F}(t)=\dot{q}_{1}(t) \cdot\left[K_{f 11} F_{1}(t)+K_{f 12} F_{2}\left(t-\tau_{2}\right)-\sum_{j=1}^{4} K_{1 d_{j}} \dot{q}_{1}(t)\right] \\
& +\dot{q}_{2}(t) \cdot\left[K_{f 11} F_{1}\left(t-\tau_{1}\right)+K_{f 12} F_{2}(t)-\sum_{j=1}^{4} K_{2 d_{j}} \dot{q}_{2}(t)\right]
\end{aligned}
$$

\section{A. Passive implementation of the PD control}

In [1] sufficient condition for passifying this part is proposed. The method is mainly based on analyzing the energy production of the controller in the frequency domain. The condition can be summarized to be as follows,

$$
K_{i d 0}=\frac{\tau_{r d}}{K_{p}} \text { for } i=1,2
$$

where $\tau_{\mathrm{rd}}$ is the round trip delay and is the sum of two delays 
$\tau_{1}$ and $\tau_{2}$

\section{B. Passive implementation of the force compensation terms}

In this section we are going to state conditions of passivity of the power associated with the force compensation terms. The $P_{f}$ is expressed in terms of both position and force signals. In order to analyze passivity one may firstly express this power term by just position signals. This can be done using the equation of the motion defined in (1). Laplace transform is applied to solve these linear differential equations,

$$
\begin{aligned}
& s M_{1} Q_{1}-K_{v}\left(e^{-\tau_{2} s} Q_{2}-Q_{1}\right)-K_{p}\left(e^{-\tau_{2} s} \frac{1}{s}\left(Q_{2}-Q_{1}\right)\right) \\
& +K_{1 d_{0}} Q_{1}+\sum_{j=1}^{4} K_{1 d_{j}} Q_{1}=\left(I+K_{f 11}\right) F_{1}+e^{-\tau_{2} s} K_{f 12} F_{2} \\
& s M_{2} Q_{2}-K_{v}\left(e^{-\tau_{1} s} Q_{1}-Q_{2}\right)-K_{p}\left(e^{-\tau_{1} s} \frac{1}{s}\left(Q_{2}-Q_{1}\right)\right) \\
& +K_{2 d_{0}} Q_{2}+\sum_{j=1}^{4} K_{2 d_{j}} Q_{1}=e^{-\tau_{1} s} K_{f 21} F_{1}+\left(I+K_{f 22}\right) F_{2}
\end{aligned}
$$

where $Q_{l}, Q_{2}$ are Laplace transforms of the truncated signals $\tilde{\dot{q}}_{1}^{t}(\theta), \tilde{\dot{q}}_{2}^{t}(\theta)$,respectively, which are defined by,

$\tilde{\dot{q}}_{i}^{t}(\theta)=[1-u(\theta-t)] \times \dot{q}_{i}(\theta), u(\lambda)$ is one if $\lambda \geq 0$ otherwise is zero. then, using these equations $F_{1}, F_{2}$ is calculated as,

$$
\begin{aligned}
& F_{1}=C_{11} \sum_{i=1}^{4} A_{i}+C_{12} \sum_{i=1}^{4} B_{i} \\
& F_{2}=C_{21} \sum_{i=1}^{4} A_{i}+C_{22} \sum_{i=1}^{4} B_{i}
\end{aligned}
$$

where $A_{i}, B_{i}$, and $C$ are defined as,

$$
\begin{aligned}
& A_{1}=s M_{1} Q_{1}, \quad A_{2}=-K_{1 v}\left(e^{-\tau_{2} s} Q_{2}-Q_{1}\right), \\
& A_{3}=-K_{1 p}\left(e^{-\tau_{2} s} \frac{1}{s}\left(Q_{2}-Q_{1}\right)\right), \quad A_{4}=\sum_{j=1}^{4} K_{1 d_{j}} Q_{1}+K_{1 d_{0}} Q_{1} \\
& B_{1}=s M_{2} Q_{2}, \quad B_{2}=-K_{2 v}\left(e^{-\tau_{1} s} Q_{1}-Q_{2}\right), \\
& B_{3}=-K_{2 p}\left(e^{-\tau_{1} s} \frac{1}{s}\left(Q_{1}-Q_{2}\right)\right), \quad B_{4}=\sum_{j=1}^{4} K_{2 d_{j}} Q_{2}+K_{2 d_{0}} Q_{2} \\
& {\left[\begin{array}{ll}
C_{11} & C_{12} \\
C_{21} & C_{22}
\end{array}\right]=\left[\begin{array}{cc}
I+K_{f 11} & e^{-\tau_{2} s} K_{f 12} \\
e^{-\tau_{1} s} K_{f 21} & I+K_{f 22}
\end{array}\right]^{-1}}
\end{aligned}
$$

Thus, human and environment force signals consists of four parts which are separated inside the sigma operator by subscript $i$. The term $i=1$ indicates the part added by the robots inertia matrix, the terms $i=2,3$ show the part added by derivative and proportional control action, and finally the term $i=4$ is the part added by dissipative actions.

Using Parseval's theorem, we can rewrite $P_{F}(t)$ in (7) as

$$
\begin{array}{r}
\int_{0}^{t} P_{F}(t) d t=\frac{1}{2 \pi} \int_{-\infty}^{+\infty} Q_{1}^{T} \cdot\left[K_{f 11} F_{1}+e^{-\tau_{2} j \omega} K_{f 12} F_{2}-\sum_{j=1}^{4} K_{1 d_{j}} Q_{1}\right] \\
+Q_{2}^{T} \cdot\left[e^{-\tau_{1} j \omega} K_{f 21} F_{1}+K_{f 22} F_{2}-\sum_{j=1}^{4} K_{2 d_{j}} Q_{2}\right] d \omega
\end{array}
$$

Clearly, the part associated with dissipative terms always dissipates energy. Thus, we will distribute this part among the other ones to passify them. otal energy associated with the force feedback can be separated considering four parts of $F_{1}$ and $F_{2}$ as

$$
\begin{aligned}
& \int_{0}^{t} P_{F}(t) d t=\frac{1}{2 \pi} \int_{-\infty}^{+\infty} Q_{1}^{T}\left[\sum_{i=1}^{4}\left(D_{11} A_{i}+D_{12} B_{i}\right)-\sum_{j=1}^{4} K_{1 d_{j}} Q_{1}\right] \\
& +Q_{2}^{T}\left[\sum_{i=1}^{4}\left(D_{21} A_{i}+D_{22} B_{i}\right)-\sum_{j=1}^{4} K_{2 d_{j}} Q_{2}\right] d \omega \\
& =\frac{1}{2 \pi} \int_{-\infty}^{+\infty} \sum_{k=1}^{3} P_{F}^{k}(\omega) d \omega
\end{aligned}
$$

where

$$
\begin{aligned}
& D_{11}=\left(K_{f 11} C_{11}+e^{-\tau_{2} j \omega} K_{f 12} C_{21}\right) \\
& D_{12}=\left(K_{f 11} C_{12}+e^{-\tau_{2} j \omega} K_{f 12} C_{22}\right) \\
& D_{21}=\left(K_{f 22} C_{21}+e^{-\tau_{1} j \omega} K_{f 21} C_{11}\right) \\
& D_{22}=\left(K_{f 22} C_{22}+e^{-\tau_{1} j \omega} K_{f 21} C_{12}\right)
\end{aligned}
$$

and the terms $P_{F}^{k}, k=1 \ldots 3$, are defined by

$$
\begin{aligned}
P_{F}^{1}= & Q_{1}^{T} \cdot\left[s D_{11} M_{1} Q_{1}+s D_{12} M_{2} Q_{2}\right] \\
& Q_{2}^{T} \cdot\left[s D_{21} M_{1} Q_{1}+s D_{22} M_{2} Q_{2}\right] \\
& -Q_{1}^{T} K_{1 d_{1}} Q_{1}-Q_{2}^{T} K_{2 d_{1}} Q_{2}=-\left\|\left[Q_{1}^{T} Q_{2}^{T}\right]^{T}\right\|_{H_{F}^{1}}
\end{aligned}
$$

where

$$
H_{F}^{1}=\left[\begin{array}{cc}
-s D_{11} M_{1}+K_{1 d_{1}} & -s D_{12} M_{2} \\
-s D_{21} M_{1} & -s D_{22} M_{2}+K_{2 d_{1}}
\end{array}\right]
$$

and

$$
\begin{aligned}
P_{F}^{2}= & -Q_{1}^{T}\left[D_{11} K_{v}\left(e^{-\tau_{2} s} Q_{2}-Q_{1}\right)+D_{12} K_{v}\left(e^{-\tau_{1} s} Q_{1}-Q_{2}\right)\right] \\
& -Q_{2}^{T}\left[D_{21} K_{v}\left(e^{-\tau_{2} s} Q_{2}-Q_{1}\right)+D_{22} K_{v}\left(e^{-\tau_{1} s} Q_{1}-Q_{2}\right)\right] \\
& -Q_{1}^{T} K_{1 d_{2}} Q_{1}-Q_{2}^{T} K_{2 d_{2}} Q_{2}=-\left\|\left[\begin{array}{ll}
Q_{1}^{T} & Q_{2}^{T}
\end{array}\right]^{T}\right\|_{H_{F}^{2}}
\end{aligned}
$$

where

$H_{F}^{2}=\left[\begin{array}{cc}D_{12} K_{v} e^{-\tau \mid s}-D_{11} K_{v}+K_{1 d_{2}} & D_{11} K_{v} e^{-\tau_{2} s_{-}} D_{12} K_{v} \\ D_{22} K_{v} e^{-\tau_{1} s}-D_{21} K_{v} & D_{21} K_{v} e^{-\tau_{2} s}-D_{22} K_{v}+K_{2 d_{2}}\end{array}\right]$

and 


$$
\begin{aligned}
P_{F}^{3}= & -Q_{1}^{T}\left[s^{-1} D_{11} K_{p}\left(e^{-\tau 2 s} Q_{2}-Q_{1}\right)+s^{-1} D_{12} K_{p}\left(e^{-\tau t s} Q_{1}-Q_{2}\right)\right] \\
& -Q_{2}^{T}\left[s^{-1} D_{21} K_{p}\left(e^{-\tau 2 s} Q_{2}-Q_{1}\right)+s^{-1} D_{22} K_{p}\left(e^{-\tau l s} Q_{1}-Q_{2}\right)\right] \\
& -Q_{1}^{T} K_{1 d 3} Q_{1}-Q_{2}^{T} K_{2 d_{3}} Q_{2}=-\left\|\left[\begin{array}{ll}
Q_{1}^{T} & Q_{2}^{T}
\end{array}\right]^{T}\right\|_{H_{F}^{3}}
\end{aligned}
$$

where

$$
H_{F}^{3}=\left[\begin{array}{cc}
\left(D_{12} K_{p} e^{-\tau \mid s}-D_{11} K_{p}\right) / s+K_{1 d_{3}} & \left(D_{11} K_{p} e^{-\tau_{2} s}-D_{12} K_{p}\right) / s \\
\left(D_{22} K_{p} e^{-\tau \mid s}-D_{21} K_{v}\right) / s & \left(D_{21} K_{p} e^{-\tau_{2} s_{2}}-D_{22} K_{p}\right) / s+K_{2 d_{3}}
\end{array}\right]
$$

and

$$
\begin{aligned}
P_{F}^{4} & =Q_{1}^{T} \cdot\left[D_{11} \sum_{j=0}^{4} K_{1 d_{j}} Q_{1}+D_{12} \sum_{j=0}^{4} K_{2 d_{j}} Q_{2}\right] \\
& +Q_{2}^{T} \cdot\left[D_{21} \sum_{j=0}^{4} K_{1 d_{j}} Q_{1}+D_{22} \sum_{j=0}^{4} K_{2 d_{j}} Q_{2}\right] \\
& -Q_{1}^{T} K_{1 d_{4}} Q_{1}-Q_{2}^{T} K_{2 d_{4}} Q_{2}=-\left\|\left[Q_{1}^{T} Q_{2}^{T}\right]^{T}\right\|_{H_{F}^{4}}
\end{aligned}
$$

where

$$
H_{F}^{4}=\left[\begin{array}{cc}
-D_{11} \sum_{j=0}^{4} K_{1 d_{j}}+K_{1 d_{4}} & -D_{12} \sum_{j=0}^{4} K_{2 d_{j}} \\
-D_{21} \sum_{j=0}^{4} K_{1 d_{j}} & -D_{22} \sum_{j=0}^{4} K_{2 d_{j}}+K_{2 d 4}
\end{array}\right]
$$

and $\|x\|_{A}=x^{T} A x$, and $s=j w$.

Now we can render the compensation part passive by making the matrices associated with each part in power terms positive definite $(P D)$. This can be done by a transformation of these matrices to the scattering matrices and ensuring that the resulting matrices have an infinity norm less than one. To clarify this lets consider one of the power terms $P_{F}^{i}$,

$$
P_{F}^{i}=-\left[\begin{array}{ll}
Q_{1}^{T} & Q_{2}^{T}
\end{array}\right] H_{F}^{i}\left[\begin{array}{l}
Q_{1} \\
Q_{2}
\end{array}\right]
$$

$P_{F}^{i}$ is the power associated with the input and output of a linear filter $H_{F}^{i}$. So, the necessary and sufficient condition for it to be passive is that the transformed scattering matrix have an infinity norm less than one [11], i.e., $\left\|S_{F}^{i}\right\| \leq 1$ where $S_{F}^{i}=\left(H_{F}^{i}-I\right)\left(H_{F}^{i}+I\right)^{-1}$ for $i=1,2$, and 3.

\section{Setting the force compensation gains}

The four force feedback gains $K_{f i j}, i, j=1,2$ have to selected appropriately in order to cancel out the effects of the force disturbances on the position coordination. Following [3], let us first decompose the system into locked and shaped systems to see the dynamic equation of the position error. Consider following state transformation
$\left(\begin{array}{l}v_{L} \\ v_{E}\end{array}\right)=\underbrace{\left(\begin{array}{cc}\mathrm{I}-\phi & \phi \\ \mathrm{I} & -\mathrm{I}\end{array}\right)}_{T}\left(\begin{array}{l}\dot{q}_{1} \\ \dot{q}_{2}\end{array}\right)$

where

$$
\phi=\left[M_{1}+M_{2}\right]^{-1} M_{2}
$$

Using this transform, the dynamics of the teleoperator system can be written as

$$
\begin{aligned}
& M_{L} \dot{\nu}_{L}=T_{L}+F_{L} \\
& M_{E} \dot{v}_{E}=T_{E}+F_{E}
\end{aligned}
$$

where $v_{E}$ is the velocity of the position error, $M_{E}, M_{L}$ are symmetric and $P D$ matrix given by

$$
\begin{aligned}
& M_{L}=M_{1}+M_{2} \\
& M_{E}=\phi^{T} M_{1} \phi+\left[\phi^{T}-\mathrm{I}\right] M_{2}[\phi-\mathrm{I}]
\end{aligned}
$$

and $T_{E}, F_{E}, T_{L}$, and $F_{L}$ are transforms for the $T_{i}, F_{i} i=1,2$ given as

$$
\left(\begin{array}{l}
T_{L} \\
T_{E}
\end{array}\right)=T^{-T}\left(\begin{array}{l}
T_{1} \\
T_{2}
\end{array}\right), \quad\left(\begin{array}{l}
F_{L} \\
F_{E}
\end{array}\right)=T^{-T}\left(\begin{array}{l}
F_{1} \\
F_{2}
\end{array}\right)
$$

To simplify the discussion suppose that in the steady state, delayed signals $q_{i}\left(t-\tau_{i}\right) i=1,2$ equal to $q_{i}(t)$. Then, by choosing $T_{E}=-F_{E}$ the effects of external forces on the position error dynamics will be compensated. This will add following terms to the control signal:

$$
\left(\begin{array}{l}
T_{1} \\
T_{2}
\end{array}\right)=T^{T}\left(\begin{array}{c}
0 \\
T_{E}
\end{array}\right)=\left(\begin{array}{c}
T_{E} \\
-T_{E}
\end{array}\right)=\left(\begin{array}{c}
F_{E} \\
-F_{E}
\end{array}\right), \quad F_{E}=\phi F_{1}+(\phi-\mathrm{I}) F_{2}
$$

so, $K_{f i j}, i, j=1,2$ are chosen as,

$$
\begin{array}{ll}
K_{f 11}=\phi & K_{f 12}=(\phi-\mathrm{I}) \\
K_{f 21}=-\phi & K_{f 22}=-(\phi-\mathrm{I})
\end{array}
$$

\section{SIMULATION}

In this section design and simulation of a 1-DOF teleoperation system is presented to illustrate the design procedure. In first step of design PD control gains are set and associated dissipation gain is set to passify it. Then in the second step after choosing appropriate gains for force compensation, other dissipation gains are set to passify these terms.

Consider a teleoperation system described by,

$$
\begin{aligned}
& \ddot{q}_{1}(t)=T_{1}(t)+F_{1}(t) \\
& \ddot{q}_{2}(t)=T_{2}(t)+F_{2}(t)
\end{aligned}
$$

where communication delays $\tau_{1}, \tau_{2}$ are chosen to be $1 \mathrm{~s}$ and $1.5 \mathrm{~s}$.

Force measurement and position and velocity signals are available at both sites. Thus, control signals are set to be in the form of Eq.4. PD gains $K_{p}$ and $K_{v}$ are set to 2 and 0.5 respectively. Using Eq.8 Dissipation term for PD gains are set to be 2.5 to passify PD action.

Now consider the first part of the power associated with the force compensation term i.e. the term $P_{F}^{1} . K_{1 f}, K_{2 f}$ are set to be $2.5 /(5+j w)$ and dissipation terms $K_{l d l}, K_{2 d l}$ are set 
to 5. As shown in Fig.2 the largest singular value of the matrix $\mathrm{S}_{\mathrm{F}}^{1}$ is less than one and consequently the chosen values render this part passive.

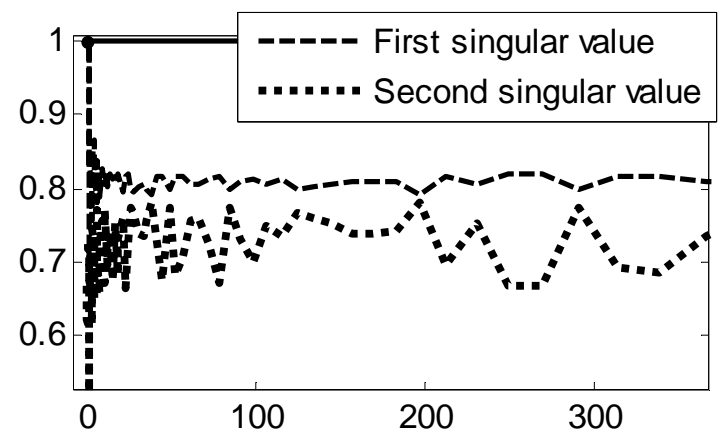

Figure.1 Singular values of the matrix $H_{F}^{1}$

The second term consists of the effects of the derivative control in the power term. The dissipation terms $K_{l d 2}, K_{2 d 2}$ are set to be 1 . This term is relatively easily passified than other terms. In Fig.3 the singular values of the corresponding scattering operator are shown.

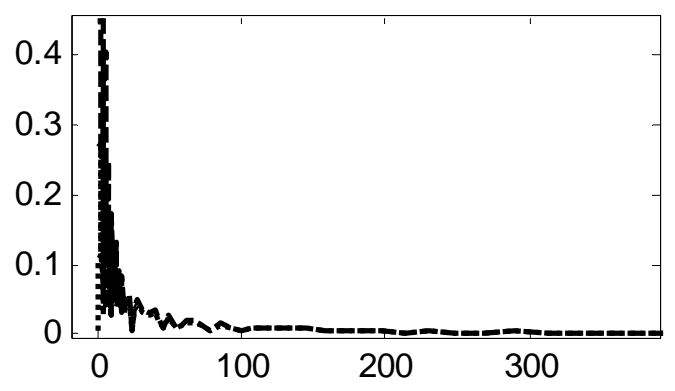

Figure.2 Singular values of the matrix $H_{F}^{2}$

For the third term $P_{F}^{3}$ dissipation terms $K_{l d 3}, K_{2 d 3}$ are chosen to be 4 . Fig. 4 depicts that with this setting the singular values of the matrix $\mathrm{S}_{\mathrm{F}}^{3}$ are both smaller than 1 .

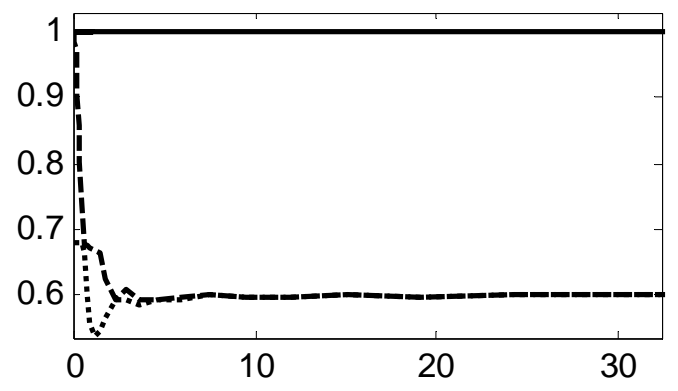

Figure.3 Singular values of the matrix $H_{F}^{3}$

Dissipation terms $K_{I d 4}, K_{2 d 4}$ are used to passify the forth power term $P_{F}^{4}$. They are set to 2.5. Fig.5 shows the passivity of this term.

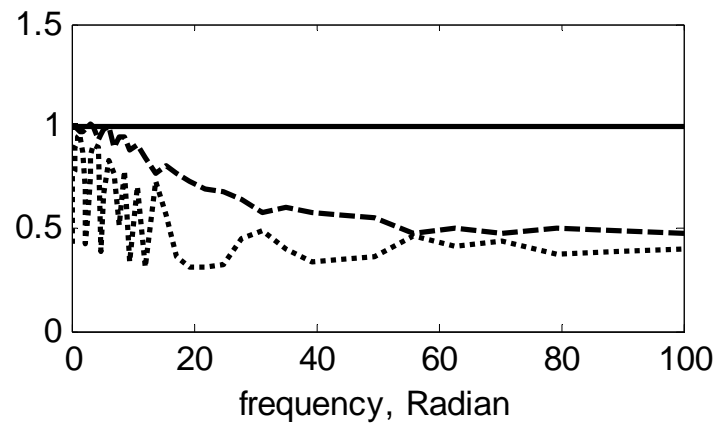

Figure.4 Singular values of the matrix $H_{F}^{4}$

To demonstrate the performance of the controller we consider the following scenario. The human operator modeled by a PD position tracking controller by its spring and damping gains as $K_{p h}$, and $K_{v h}$ respectively. Firstly, he pushes the master robot to the position 0.8 . Then, he tries to push the master robot to the position 1.5. While he is moving the robot to this target, he realizes the existence of a hard wall while receiving step-like force feedback. At the steady state motion of pushing this wall, we will have position coordination as well as force tracking and this point is the main contribution of this paper. Finally, he pushes the master robot back to its initial position. Chosen and designed values for the simulation are summarized in Table.1. Simulation results are shown in Fig.5.

\begin{tabular}{cc|lc}
\hline \multicolumn{4}{c}{ Table.1. Parameters in the simulation } \\
\hline $\begin{array}{c}\text { Chosen } \\
\text { parameter }\end{array}$ & Value & $\begin{array}{l}\text { Designed } \\
\text { parameter }\end{array}$ & Value \\
$\tau_{1}$ & $1 \mathrm{~s}$ & $K_{l d 0}=K_{2 d 0}$ & 5 \\
$\tau_{2}$ & $1.5 \mathrm{~s}$ & $K_{l d l}=K_{2 d l}$ & 5 \\
$M_{l}=M_{2}$ & 1 & $K_{l d 2}=K_{2 d 2}$ & 1 \\
$K_{l p}=K_{2 p}$ & 1 & $K_{l d 3}=K_{2 d 3}$ & 4 \\
$K_{l v}=K_{2 v}$ & 0.5 & $K_{l d 4}=K_{2 d 4}$ & 2.5 \\
$K_{l F}=K_{2 F}$ & $2.5 /(5+\mathrm{s})$ & &
\end{tabular}

\section{CONCLUSION}

A control framework for control of a teleoperation system in the presence of large time delay was presented. This framework provides position coordination in both cases of free and contact motions while preserves passivity of the teleoperator. Pre-specified simple structure of the controller presents low cost and easily implemented control framework. Control system is separated to position and force feedback parts. The passification of the first part is studied in [1] by Lee et al. To compensate the environment forces and, hence, achieving position tracking in contact tasks force feedbacks are also added to the control system. The effects of this part on total energy are analyzed and sufficient condition for its passivity is obtained through studying the effects of several parts this force feedback adds. To 

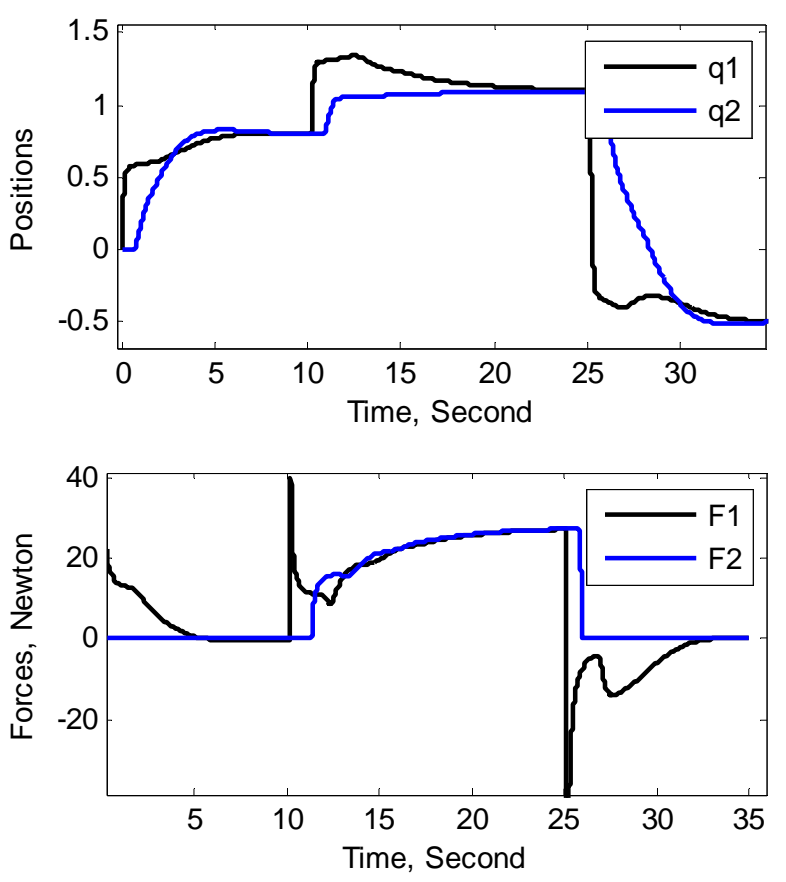

Figure.5 Results of the simulation

demonstrate how velocity feedbacks are applied to passify the overall control system an illustrative example are also presented. Next research projects may consist of extending the results to the case of the teleoperation of nonlinear robots.

\section{REFERENCES}

[1] D. Lee \& M. W. Spong, "Passive bilateral teleoperation with constant time delay," IEEE Transactions on Robotics, v22, n2, p.269-281, 2006.

[2] D. J. Lee and P. Y. Li, "Passive bilateral control and tool dynamics rendering for nonlinear mechanical teleoperators," IEEE Trans. Robot., vol.21, no. 5, pp.936-951, Oct. 2005.

[3] D. J. Lee and P. Y. Li, "Passive bilateral feedforward control of linear dynamically similar teleoperated manipulators," IEEE Trans. Robot. Autom., vol. 19, no.3, pp. 443-456, Jun 2003

[4] D. Lawrence, "Stability and Transparency in Bilateral Teleoperation", IEEE Trans. Robot. Autom., vol. 9, no.3, pp.624-637, 1993.

[5] Mc. Cavusoglu, A. Sherman, and F. Tendick, "Design of bilateral teleoperation controllers for haptic exploration and telemanipulation of soft environment," IEEE transactions on robotic and automation, August 2002.

[6] S. Sirouspour, "Modelling and control of cooperative teleoperation systems," IEEE Transaction on Robotics, vol. 21, no. 6, Dec. 2005, pp. $1220-1225$

[7] T.J. Tarn, Y.Y. Wu, N. Xi and A. Isidori, "Force Regulation and Contact Transition Control," IEEE Control Systems Magazine, Feb. 1996.

[8] Liya Ni and David W. L. Wang, "A Gain-Switching Control Scheme for Position-Error-Based Bilateral Teleoperation: Contact Stability Analysis and Controller Design," The International Journal of Robotics Research, pp. 255-274, 2004.

[9] D. T. Surdilovic, "Contact Transition Stability in the Impedance Control," IEEE, ICRA, Apr, 1997.

[10] G. Niemeyer and J. J.Slotine, "Stable Adaptive Teleoperation," IEEE Journal of Oceanic Engineering, vol.16, no.1, pp.152-162, 1991.

[11] R.J. Anderson and M.W. Spong, "Bilateral control of operators with time delay," IEEE Trans. Automat. Cont., vol. AC-34,pp. 494\{501, May 1989.

[12] N. Hogan, "On the Stability of Manipulators Performing Contact Task," J. of Robotics and Automation, 4-6, pp.677-686, 1988.

[13] G.M.H. Leung, B.A. Francis, and J. Apkarian, "Bilateral controller for teleoperators with time delay via $\mu$ synthesis, " January 1995.

[14] J.E. Colgate, "Robust Impedance Shaping Telemanipulation," IEEE Transactions on Robotics and Automation, vol. 9, pp. 374-384, August 1993.

[15] J. Yan and S. Salcudean, "Teleoperation controller design using Hinfinity Optimization with application to motion-scaling," IEEE Trans.Contr. Syst. Technol., vol. 45, no. 2, pp. 244-258, May 1996.

[16] K. Hashtrudi-Zaad and S.E. Salcudean, "Analysis and Evaluation of Stability and Performance Robustness for Teleoperation Control Archetectures," IEEE Int. Conf. on Robotics and Auto., San Francisco, CA, April 